\title{
Improvement in patient-reported outcomes after lung transplantation is not impacted by the use of extracorporeal membrane oxygenation as a bridge to transplantation

Nicholas A. Kolaitis, MD, ${ }^{\mathrm{a}}$ Allison Soong, BS, MPH, ${ }^{\mathrm{a}}$ Pavan Shrestha, BA, ${ }^{\mathrm{a}}$ Hanjing Zhuo, MD, MPH, ${ }^{\mathrm{a}}$ John Neuhaus, PhD, ${ }^{b}$ Patti P. Katz, PhD, ${ }^{\mathrm{c}}$ John R. Greenland, MD, PhD, ${ }^{\mathrm{a}}$ Jeffrey Golden, MD, Lorriana E. Leard, MD, ${ }^{\mathrm{a}}$ Rupal J. Shah, MD, MSCE, ${ }^{\mathrm{a}}$ Steven R. Hays, MD, ${ }^{\mathrm{a}}$ Jasleen Kukreja, MD, ${ }^{\mathrm{d}}$ Mary Ellen Kleinhenz, MD, ${ }^{\mathrm{a}}$ Paul D. Blanc, MD, MSPH, ${ }^{\mathrm{a}}$ and Jonathan P. Singer, MD, MS

\section{ABSTRACT}

Objective: Extracorporeal membrane oxygenation (ECMO) is increasingly used as a bridge to lung transplantation. The impact of preoperative ECMO on healthrelated quality of life (HRQL) and depressive symptoms after lung transplantation remains unknown, however.

Methods: In a single-center prospective cohort study, we assessed HRQL and depressive symptoms before and at 3, 6, and 12 months after lung transplantation using the Short Form 12 Physical and Mental Component Scores (SF12-PCS and SF12-MCS), Airway Questionnaire 20-Revised (AQ20R), EuroQol 5D (EQ5D), and Geriatric Depression Scale (GDS). Changes in HRQL were quantified by segmented linear mixed-effects models controlling for age, sex, diagnosis, preoperative forced expiratory volume in 1 second, 6-minute walk distance, and Lung Allocation Score. We compared changes in HRQL among subjects bridged with ECMO, subjects hospitalized but not on ECMO, and subjects called in for transplantation as outpatients.

Results: Out of 189 subjects, 17 were bridged to transplantation with ECMO. In all groups, improvements in HRQL following lung transplantation exceeded the minimally clinically important difference using the SF12-PCS, AQ20R, EQ5D, and GDS. HRQL defined by SF12-MCS did not change after transplantation. Improvements were generally similar among the groups, except for EQ5D, which showed a trend toward less benefit in the outpatients, possibly due to their better HRQL before lung transplantation.

Conclusions: Subjects ill enough to require ECMO as a bridge to lung transplantation appear to achieve similar improvements in HRQL and depressive symptoms as those who do not. It is reassuring to both providers and patients that lung transplantation provides substantial improvements in HRQL, even for those patients who are critically ill in the run up to transplantation. (J Thorac Cardiovasc Surg 2018;156:440-8)

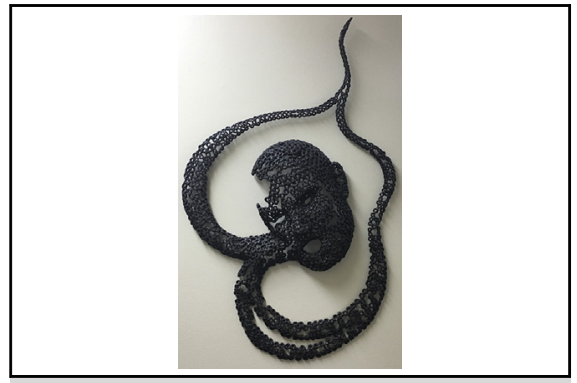

Metal sculpture made by a survivor of ECMO as a bridge to lung transplantation.

\section{Central Message}

The impact of lung transplantation on healthrelated quality of life and depressive symptoms for patients requiring preoperative extracorporeal membrane oxygenation is large and similar to that seen in noncritically ill transplant candidates.

\section{Perspective}

Lung transplantation improves health-related quality of life and depressive symptoms for patients with end-stage lung disease. Patients requiring extracorporeal membrane oxygenation (ECMO) before transplantation achieve the same magnitude of improvement as noncritically ill transplantation candidates. This knowledge provides clinicians more tools to counsel critically ill patients when considering ECMO as a bridge to transplantation.

See Editorial Commentary page 449.

See Editorial page 438 .
From the ${ }^{\mathrm{a}}$ Division of Pulmonary and Critical Care, Department of Medicine, ${ }^{\mathrm{b}} \mathrm{De}-$ partment of Epidemiology and Biostatistics, ${ }^{\mathrm{c}}$ Division of Rheumatology, Department of Medicine, and Division of Thoracic Surgery, Department of Surgery, University of California San Francisco School of Medicine, San Francisco, Calif. Funding was provided by the National Heart, Lung and Blood Institute (Grant K23 HL111115, to J.P.S.)

Received for publication Sept 12, 2017; revisions received Jan 8, 2018; accepted for publication Jan 20, 2018; available ahead of print March 14, 2018.
Address for reprints: Nicholas A. Kolaitis, MD, Division of Pulmonary and Critical Care, Department of Medicine, University of California San Francisco, 400 Parnassus Ave, Box 0359, San Francisco, CA 94143 (E-mail: nicholas.kolaitis@ucsf. edu).

$0022-5223 / \$ 36.00$

Copyright $(2018$ by The American Association for Thoracic Surgery https://doi.org/10.1016/j.jtcvs.2018.01.101 

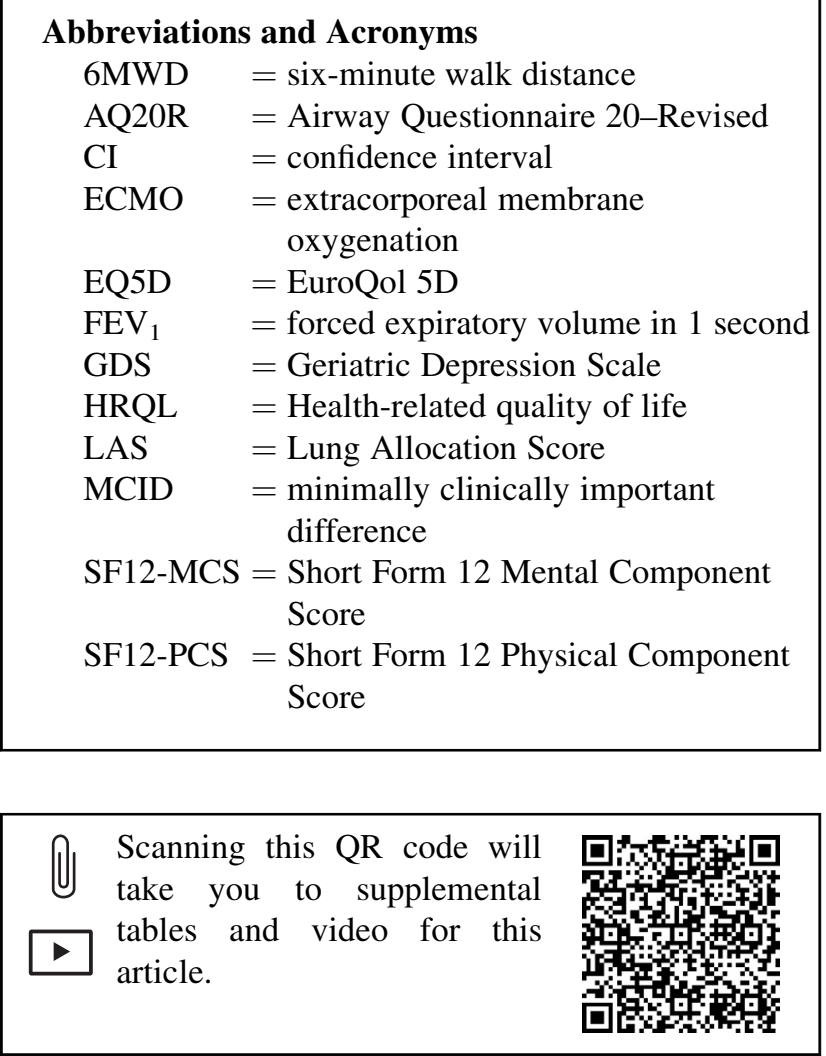

Lung transplantation aims to extend survival and to improve health-related quality of life (HRQL) for patients suffering from end-stage lung disease. ${ }^{1-3}$ Historically, many lung transplant programs have been reluctant to offer transplantation to critically ill patients not only because of concern for poorer short-term survival, but also due to compromised functional status and quality of life in those who do survive. ${ }^{4-7}$ With increasing experience in the use of extracorporeal membrane oxygenation (ECMO) as a bridge to transplantation, critically ill patients are more likely to be offered the opportunity for lung transplantation. $^{8}$ Although the first successful case of ECMO as a bridge to lung transplantation was reported in 1977 , this practice remained relatively uncommon, with only 24 patients treated in this manner in the United States through $2000 .^{9}$ From 2000 to 2014, however, the number of cases rose to 414 , a remarkable 28 -fold increase. ${ }^{10}$

Current evidence supports not only that bridging patients to lung transplantation with ECMO is feasible, but also that survival after transplantation may be comparable to that in individuals who undergo transplantation while critically ill but not on ECMO ${ }^{11-14}$ Understanding the impact of lung transplantation on $\mathrm{HRQL}$ in this rapidly growing population of candidates is fundamental to defining the benefit of transplantation.
Preoperative ECMO is associated with a lower likelihood of survival to lung transplantation, increased cost of hospitalization, and, if transplantation is performed, a longer postoperative length of stay. ${ }^{15-17}$ ECMO also carries specific additional risks, including hemorrhage, hemolysis, neurologic injury, limb ischemia, and sepsis. ${ }^{18}$ The complications related to critical illness and ECMO are known to have a negative impact in nontransplantation populations; for example, survivors of general critical illness have poorer physical functioning than healthy controls and have a high incidence of depressive symptoms. ${ }^{19,20}$ Furthermore, those who survive critical illness with the assistance of ECMO report poor subsequent HRQL and persistent physical and emotional health limitations. ${ }^{21-23}$ Because surviving to lung transplantation through ECMO might be similar to surviving critical illness, it is plausible that the impact of lung transplantation on HRQL benefit may be attenuated in this group.

Given the possibility that bridging to lung transplantation with ECMO may attenuate HRQL improvements, we sought to evaluate whether the impact of lung transplantation on HRQL within the first postoperative year was different in patients who were bridged to transplantation with ECMO compared with those who were not. To do so, we performed a single-center prospective cohort study using multiple instruments to assess HRQL, including generic, respiratory-specific, and health utility instruments. Given the high incidence of depression following critical illness, we also measured depressive symptoms with a non-HRQL instrument.

\section{METHODS}

\section{Study Design, Participants, and Setting}

We performed this study among participants in the University of California San Francisco's "Breathe Again" study. The details of the study design, including exclusion criteria, have been described previously. ${ }^{3}$ In brief, Breathe Again is a prospective cohort study of subjects age $\geq 18$ years who underwent first-time lung transplantation between February 2010 and January 2017; the follow-up reported here continued until May 2017.

Subjects were enrolled at the time of placement on the lung transplant waiting list. At enrollment, subjects underwent a research visit that included functional assessments as well as a battery of HRQL measures and a depression scale. While subjects were on the wait list, assessments were repeated every 3 months or at the time of major changes in clinical status, including terminal hospitalization requiring transplantation, need for invasive mechanical ventilation, or implementation of ECMO. The same functional and HRQL assessments were administered at 3,6, and 12 months after transplantation. Our study cohort comprised a total of 189 subjects (Figure 1).

At our center, ECMO is used in patients with worsening clinical status that leads to cor pulmonale and/or profound hypoxemia with oxygen needs exceeding what can be provided by mechanical ventilation. ECMO is used both in patients already listed for lung transplantation and in patients needing extra support to complete their evaluation for transplantation (Video 1). Patients eligible for ECMO must be under age 65 years. ECMO is typically deployed in an internal jugular dual lumen venovenous 
Subjects Enrolled in Study and Underwent

Transplant During Study Period

$\mathrm{n}=254$

\section{Reasons for missing survey}

Subjects who underwent transplant < age 65

(not eligible for ECMO if $>65$ years old)

$\mathrm{n}=189 ; 167$ surveys completed

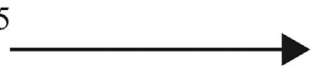

13 too ill to complete

9 non-health reasons

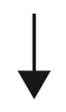

3 months post transplant (stopped collecting 3 month survery July 2016) $\mathrm{n}=189 ; 144$ surveys completed

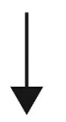

6 months post transplant $\mathrm{n}=186 ; 145$ surveys completed

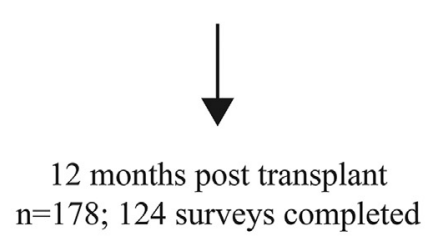

12 months post transplant $\mathrm{n}=178 ; 124$ surveys completed
2 died

6 too ill to complete

9 non-health reasons

1 dropped out

3 study ended

24 three month survey discontinued

2 died

7 too ill to complete

23 non-health reasons

2 dropped out

7 study ended

5 died

6 too ill to complete

24 non-health reasons

3 dropped out

16 study ended

FIGURE 1. Flow chart of subjects throughout the study. The left column presents the number of subjects providing data for analysis at each time point. The number of subjects at the 3-month posttransplantation time point was less than 6-month time point, because we stopped administering surveys at 3 months posttransplantation after July 2016. The right column explains the reasons for missing surveys at each time point. ECMO, Extracorporeal membrane oxygenation.

cannula placement, unless the patient has pulmonary hypertension, with pulmonary artery systolic pressure $>50 \mathrm{~mm} \mathrm{Hg}$. When patients have pulmonary hypertension, peripheral or central venoarterial ECMO is used. Since we began our ECMO as a bridge to transplantation program in 2004, 61 patients have been placed on ECMO with the intent of facilitating survival to lung transplantation. Of these 61 patients, $52(85 \%)$ were successfully bridged to lung transplantation. Seventeen of these patients were enrolled as subjects in the Breathe Again cohort.

Because our center does not offer ECMO to patients age $>65$ years, we restricted our analysis to Breathe Again study participants age $\leq 65$ years. We defined 3 groups: subjects bridged to lung transplantation with ECMO, subjects hospitalized but not on ECMO at the time of transplantation, and subjects called in for transplantation as outpatients. This study was approved by the Committee on Human Research at the University of California San Francisco (protocol no. 10-04020; approved December 3, 2010), and written informed consent was obtained from all study participants. Some of these data have been presented previously. ${ }^{3,24}$

\section{Outcome Variables: HRQL and Depressive Symptoms}

HRQL is, by definition, multidimensional. ${ }^{25,26}$ Existing HRQL instruments emphasize different conceptual health domains relevant to the population for which they were developed. Because various instruments provide complementary information, we used multiple

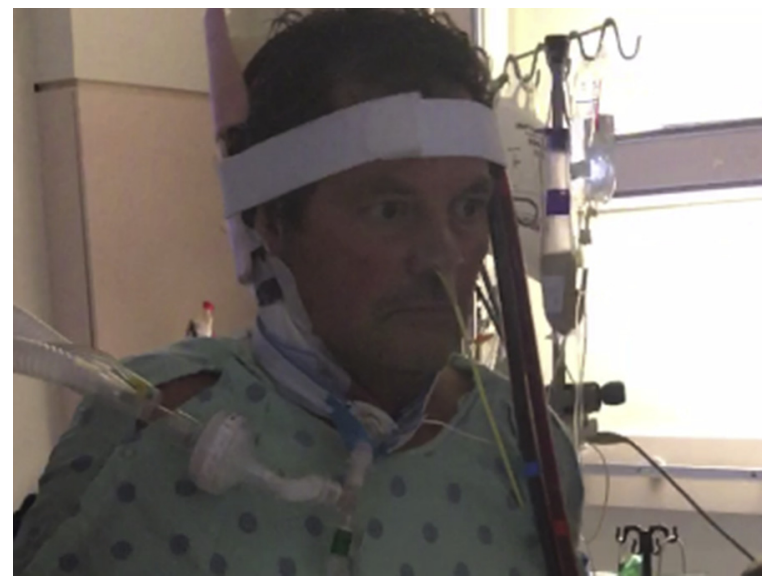

VIDEO 1. Extracorporeal membrane oxygenation (ECMO) as a bridge to lung transplantation. Video of a University of California San Francisco lung transplant recipient with idiopathic pulmonary fibrosis who was placed on ECMO as a bridge to transplantation. With ECMO, the patient was able to ambulate and maintain his strength before transplantation despite hypoxemic respiratory failure. Video available at: http://www. jtcvsonline.org/article/S0022-5223(18)30575-0/fulltext. 
measures to more comprehensively measure conceptual HRQL domains relevant to lung transplantation (Table E1). Specifically, we measured HRQL and depressive symptoms at pretransplantation baseline and at 3, 6, and 12 months after transplantation. To evaluate generic HRQL, we used the Medical Outcomes Survey Short Form 12, version 2, Physical and Mental Component Summary scores (SF12-PCS and SF12-MCS; range, $0-100$, with higher scores indicating better HRQL). ${ }^{27}$ A change of 5 points in either of these measures is generally considered to represent a minimally clinically important difference (MCID). ${ }^{28}$ To evaluate respiratory-specific HRQL, we used the Airways Questionnaire 20Revised (AQ20-R; range 0-20, with lower scores indicating better HRQL; 0.5 standard deviation used as distribution-based MCID: 1.75). ${ }^{29,30}$ To evaluate health utility, we used the EuroQoL 5D (EQ5D; range, -0.11 to 1.0 , with higher scores indicating better HRQL and a score $<0$ reflecting a state "worse than death"; MCID: 0.06). ${ }^{3}$

It is estimated that as many as $63 \%$ of organ transplant recipients experience symptoms of depression or anxiety within the first few years after transplantation. ${ }^{32}$ Depressive symptoms following transplantation are
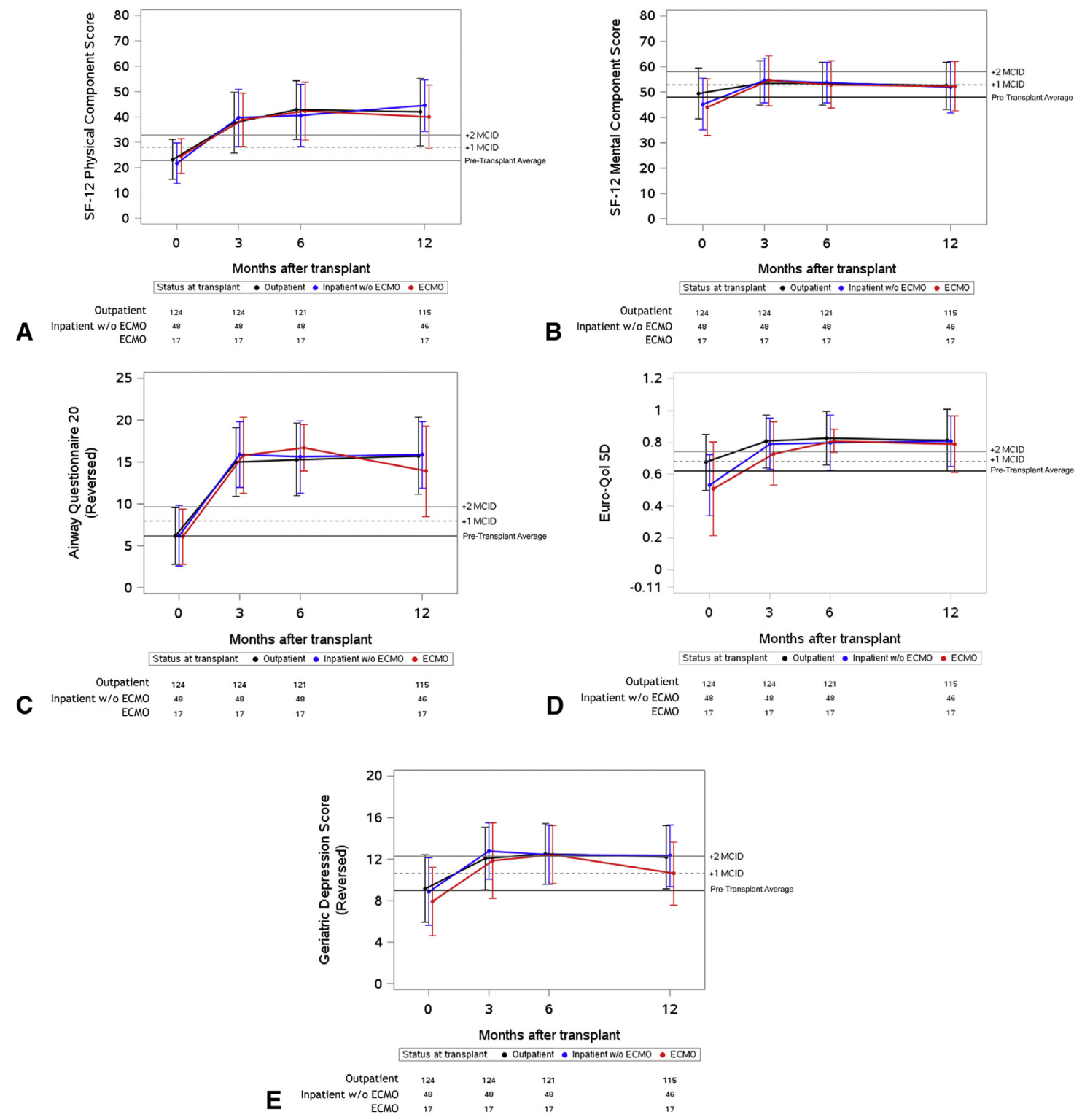

FIGURE 2. Unadjusted plots of average health-related quality of life (HRQL) from before to 1 year after lung transplantation. A, Short Form 12 Physical Component Score (SF-12PCS; generic-physical HRQL). B, Short Form 12 Mental Component Score (SF-12MCS; generic-mental HRQL). C, Airway Questionnaire 20-Revised (AQ20R; respiratory-specific HRQL). D, EuroQol 5D (EQ5D; health utility HRQL). E, Geriatric Depression Scale (GDS). Red lines represent subjects who required ECMO as a bridge to transplantation, blue lines represent subjects who were hospitalized but not on ECMO at the time of transplantation, and black lines represent subjects called in for transplantation from the outpatient setting. Whiskers represent 1 standard deviation above and below the mean HRQL value for each time point. On the $y$-axis, the first horizontal black line denotes pretransplantation mean scores, and the dashed and solid horizontal lines above denote a change in score equal to 1 time and 2 times the minimally clinically important difference, respectively. $M C I D$, Minimally clinically important difference; ECMO, extracorporeal membrane oxygenation. 
TABLE 1. Cohort demographics and clinical variables

\begin{tabular}{|c|c|c|c|c|c|}
\hline Variable & $\begin{array}{l}\text { ECMO group } \\
(\mathbf{n}=\mathbf{1 7})\end{array}$ & $\begin{array}{l}\text { Inpatient group } \\
\quad(n=48)\end{array}$ & $\begin{array}{l}\text { Outpatient group } \\
\quad(\mathbf{n}=\mathbf{1 2 4})\end{array}$ & $\begin{array}{c}\text { Whole cohort } \\
\quad(n=189)\end{array}$ & $P$ value \\
\hline Age, y, mean $\pm S D$ & $46.1 \pm 16.6$ & $52.9 \pm 9.0$ & $51.8 \pm 11.6$ & $51.6 \pm 11.6$ & .11 \\
\hline Female sex, n $(\%)$ & $9(53)$ & $24(50)$ & $52(42)$ & $85(45)$ & \\
\hline Male sex, n (\%) & $8(47)$ & $24(50)$ & $72(58)$ & $104(55)$ & .50 \\
\hline White, n (\%) & $12(80)$ & $26(59)$ & $84(70)$ & $122(68)$ & \\
\hline Black/African American, n (\%) & $1(7)$ & $5(11)$ & $11(9)$ & $17(9)$ & \\
\hline Asian, $n(\%)$ & $0(0)$ & $2(5)$ & $10(8)$ & $12(7)$ & \\
\hline Hispanic, n (\%) & $2(13)$ & $9(20)$ & $16(13)$ & $27(15)$ & \\
\hline Hawaiian/Pacific Islander, $\mathrm{n}(\%)$ & $0(0)$ & $1(2)$ & $0(0)$ & $1(1)$ & \\
\hline Other, n $(\%)$ & $0(0)$ & $1(2)$ & $0(0)$ & $1(1)$ & .42 \\
\hline LAS group A: COPD, n (\%) & $1(6)$ & $4(8)$ & $21(17)$ & $26(14)$ & \\
\hline LAS group B: PAH, n (\%) & $1(6)$ & $1(2)$ & $8(6)$ & $10(5)$ & \\
\hline LAS group C: CF, n (\%) & $2(11)$ & $4(8)$ & $17(14)$ & $23(12)$ & \\
\hline LAS group D: IPF, n (\%) & $13(76)$ & $39(81)$ & $78(63)$ & $130(69)$ & .36 \\
\hline Expired at $1 \mathrm{y}, \mathrm{n}(\%)$ & $1(6)$ & $2(4)$ & $2(2)$ & $5(3)$ & .44 \\
\hline $\mathrm{FEV}_{1}, \mathrm{~L}$, mean $\pm \mathrm{SD}$ & $1.7 \pm 0.77$ & $1.35 \pm 0.74$ & $1.41 \pm 0.70$ & $1.43 \pm 0.72$ & .17 \\
\hline $\mathrm{FVC}, \mathrm{L}$, mean $\pm \mathrm{SD}$ & $2.29 \pm 0.74$ & $1.84 \pm 0.89$ & $2.07 \pm 0.78$ & $2.03 \pm 0.81$ & .10 \\
\hline BMI, $\mathrm{kg} / \mathrm{m}^{2}$, mean $\pm \mathrm{SD}$ & $24.5 \pm 4.9$ & $25.3 \pm 4.1$ & $25.4 \pm 4.4$ & $25.3 \pm 4.4$ & .75 \\
\hline $6 \mathrm{MWD}, \mathrm{m}$, mean $\pm \mathrm{SD}$ & $212 \pm 199$ & $176 \pm 133$ & $283 \pm 126$ & $250 \pm 143$ & $<.001$ \\
\hline LAS, mean \pm SD & $67.8 \pm 26.6$ & $69.2 \pm 19.4$ & $44.5 \pm 11.4$ & $52.8 \pm 19.3$ & $<.001$ \\
\hline
\end{tabular}

Diagnostic indication for transplantation was categorized by the A (COPD), B (PAH), C (Cystic Fibrosis), D (IPF) groupings in the Lung Allocation Score. ${ }^{37}$ ECMO, Extracorporeal membrane oxygenation; $S D$, standard deviation; $L A S$, Lung Allocation Score; $C O P D$, chronic obstructive pulmonary disease; $P A H$, pulmonary arterial hypertension; $C F$, cystic fibrosis; $I P F$, idiopathic pulmonary fibrosis; $F E V_{l}$, forced expiratory volume in 1 second; $F V C$, forced vital capacity; $B M I$, body mass index; $6 M W D, 6$-minute walk distance.

also strongly associated with subsequent mortality. ${ }^{32,33}$ Similarly, survivors of critical illness experience high rates of depression. ${ }^{34}$ Thus, we were interested in whether subjects bridged with ECMO would report more depressive symptoms compared with other transplant recipients. Because HRQL instruments include only a limited number of items addressing depressive symptoms, we were not able to use a validated HRQL instrument to assess subjects for depressive symptoms. Thus, we used the short form of the Geriatric Depression Scale (GDS; range, 0-15, with lower scores indicating fewer depressive symptoms; 0.5 standard deviation used as distribution-based MCID: 1.65). ${ }^{30,35}$

\section{Other Variables}

Baseline demographic and clinical variables were abstracted from our lung transplant center medical records. Variables collected included age, sex, diagnostic indication for transplantation as categorized by the Lung Allocation Score (LAS), ${ }^{36}$ preoperative forced expiratory volume in 1 second $\left(\mathrm{FEV}_{1}\right.$, in liters), 6-minute walk distance (6MWD, in meters), and the LAS. For this analysis, we considered the data closest to the date of transplantation.

\section{Analytic Approach}

Baseline continuous and categorical variables were compared among our 3 groups using analysis of variance and the $\chi^{2}$ test, as appropriate. For the SF12-PCS, SF12-MCS, and EQ5D, higher scores denote better HRQL. Lower scores denote better HRQL for the AQ20R and less depressive symptoms for the GDS. To facilitate interpretation, we reverse-coded the AQ20R and GDS, so that higher scores would represent better HRQL by AQ20R and less depressive symptoms by GDS. Changes in HRQL scores were plotted over time (Figure 2). Because our primary outcome was to quantify the magnitude of change in HRQL from before transplantation to after transplantation between groups, we did not compare the individual absolute scores between groups at the 3-, 6-, and 12-month time points. Visual inspection of the change in HRQL over time demonstrated 2 distinct periods with a different slope of change in HRQL; the early period of change from pretransplantation baseline to 6 months posttransplantation (which accounted for most of the change in HRQL), and the late period of change from 6 to 12 months posttransplantation (which had a relatively flat slope for all instruments).

Because the change in HRQL over time was not linear, changes in HRQL and depressive symptoms over time were estimated using segmented linear mixed-effects models with a joint point set at 6 months after transplantation. Subject and time were included as random effects, allowing for different slopes in the HRQL outcomes in the early and later time periods within each subject. Parameter estimates and the $95 \%$ confidence intervals (CIs) for the other groups were derived by postestimation commands (lincom). For each outcome, we performed unadjusted analyses as well as analyses adjusted for age, sex, diagnostic category, most recent preoperative $\mathrm{FEV}_{1}$ value, most recent preoperative 6MWD, and LAS. Since depressive symptoms are strongly associated with general HRQL, we also performed a secondary analysis in which we controlled for preoperative GDS. For all models, we tested whether HRQL or GDS changed from baseline to after transplantation within each group and whether the magnitude of the change was different across groups. Since our primary aim was to estimate the within-subject change in HRQL across all time points (including baseline), we did not control for baseline HRQL.

Not all subjects completed all study visits. Overall, the number of missing surveys was small (104 of 742 potential timepoints; $14 \%$ ). We did not impute these missing data, because the maximum likelihood methods used to fit mixed-effects models provide valid estimates for 
TABLE 2. Health-related quality of life before lung transplantation

\begin{tabular}{lcccc}
\hline \multicolumn{1}{c}{ Instrument } & $\begin{array}{c}\text { ECMO group } \\
(\mathbf{n}=\mathbf{1 7})\end{array}$ & $\begin{array}{c}\text { Inpatient group } \\
(\mathbf{n}=\mathbf{4 8})\end{array}$ & $\begin{array}{c}\text { Outpatient group } \\
(\mathbf{n}=\mathbf{1 2 4})\end{array}$ & $\begin{array}{c}\text { Whole cohort } \\
(\mathbf{n}=\mathbf{1 8 9})\end{array}$ \\
\hline SF12-PCS (MCID = 5) & $24.7 \pm 6.8$ & $21.7 \pm 8.0$ & $23.3 \pm 7.9$ & $23.0 \pm 7.8$ \\
SF12-MCS (MCID = 5) & $43.9 \pm 11.1$ & $45.3 \pm 10.0$ & $49.5 \pm 9.9$ & .35 \\
AQ20R (MCID = 1.75) & $6.1 \pm 3.3$ & $6.2 \pm 3.7$ & $6.2 \pm 3.4$ & .01 \\
EQ5D (MCID = 0.06) & $0.51 \pm 0.29$ & $0.53 \pm 0.19$ & $0.68 \pm 0.17$ & $6.2 \pm 3.4$ \\
GDS (MCID = 1.65) & $7.9 \pm 3.3$ & $8.9 \pm 3.3$ & $9.2 \pm 3.3$ & $0.62 \pm 0.21$ \\
\hline
\end{tabular}

Data are presented as unadjusted mean score \pm standard deviation. Health-related quality of life instruments: generic physical instrument: SF12-PCS (Short Form 12-Physical Component Score), range 0 to 100; generic mental instrument: SF12-MCS (Short Form 12-Mental Component Score), range 0 to 100; respiratory-specific instrument: AQ20R (Airways Questionnaire 20-Revised), range 0 to 20, reverse-coded for analysis; health-utility instrument: EQ5D (EuroQoL 5D), range - 1.11 to 1; depression scale: GDS (Geriatric Depression Scale), range 0 to 15, reverse-coded for analysis. ECMO, Extracorporeal membrane oxygenation; SF12-PCS, short form 12-physical component score; $M C I D$, minimally clinically important difference; SF12-MCS, short form 12-mental component score; AQ20R, airways questionnaire 20-revised; EQ5D, EuroQoL 5D; GDS, geriatric depression scale.

missing values. ${ }^{37}$ Nevertheless, it is possible that informative missingness could have impacted our effect estimates. Consequently, we performed a sensitivity analysis in which we imputed missing values. Missing data were considered missing at random if a subject did not complete the survey for reasons other than health (eg, missed the clinic appointment because of inclement weather, left the clinic before completing the survey because of traffic). If data were believed to be missing at random, the clinical chart was reviewed to ensure that the subject had stable allograft function and was not dealing with an acute medical issue. A survey was categorized as missing not at random if subject was too ill to complete the survey. For data missing at random, we used 10-fold multiple imputation with chained equations. ${ }^{38}$ For data missing not at random, we imputed scores using the median of the lowest quartile of HRQL scores for all other participants at that time point, as we have done previously. ${ }^{3}$ Analyses were conducted using Stata 15 (StataCorp, College Station, Tex).

\section{RESULTS}

Of the 259 subjects who completed pretransplantation assessments and underwent transplantation during the study period, the 189 subjects age $<65$ composed the cohort for this analysis (Figure 1). Of this cohort, 17 subjects $(9 \%)$ were on ECMO support at the time of transplantation, 48 $(25 \%)$ were inpatients but not on ECMO, and $124(66 \%)$ were outpatients. The patients placed on ECMO as a bridge to transplantation included 8 placed on venoarterial ECMO and 9 placed on venovenous ECMO. Ten of the patients were listed at the time of being placed on ECMO, and 7 of the patients were placed on ECMO to facilitate completion of lung transplant evaluation. In addition, 3 of the patients bridged to transplantation on ECMO required prolonged ECMO support after transplantation. In the entire cohort, 183 subjects underwent bilateral lung transplantation (including all those who were placed on ECMO as a bridge to transplantation), 3 underwent single lung transplantation, and 3 underwent combined heart and lung transplantation. The 3 groups were similar in terms of age, sex, race, body mass index, pretransplantation disease category, $\mathrm{FEV}_{1}$, and forced vital capacity (FVC) (Table 1). The groups had significant differences in 6MWD and LAS $(P<.001)$.

Overall survival at 1 year after transplantation was $97 \%$ and was similar in the 3 groups $(P=.44)$. One subject in the
ECMO group $(1 / 17 ; 6 \%), 2$ subjects in the inpatient but not on ECMO group $(2 / 48 ; 4 \%)$, and 2 subjects in the outpatient group $(2 / 124 ; 2 \%)$ died within the first year.

Before transplantation, HRQL was similar among the 3 groups as measured by the SF12-PCS and AQ20R. Depressive symptoms as assessed by the GDS were also similar among the 3 groups (Table 2). Outpatients reported better baseline HRQL by the SF12-MCS and EQ5D. After transplantation, HRQL and depressive symptoms generally improved across all 3 groups (Figure 2). Overall, peak improvement in HRQL and depressive symptoms was seen in the early period, within 6 months posttransplantation, and remained stable in the late period, through 12 months posttransplantation. The magnitude of these early improvements varied by instrument, however (Table 3). For example, in generic physical HRQL (SF12PCS), early improvements exceeded 3 times the MCID for all groups $(P$ value for change $<.001 ; P$ value for difference among groups $=.27$ ).

All groups reported early improvement in respiratoryspecific HRQL, exceeding 5 times the MCID ( $P$ value for change $<.001 ; P$ value for difference among groups $=.59)$. In health utility $(\mathrm{EQ} 5 \mathrm{D})$, both the ECMO and inpatient groups showed early improvements of 4 times the MCID, whereas the outpatient group improved by nearly 3 times the MCID $(P$ value for change $<.001 ; P$ value for difference among groups $=.001$ ). Of note, the baseline EQ5D scores were higher in the outpatient group, whereas after transplantation, the absolute EQ5D scores were similar across the 3 groups. Depressive symptoms exhibited early improvements that exceeded the MCID in all groups $(P$ value for change $\leq .001 ; P$ value for difference among groups $=.09$ ). An exception to the improvement in HRQL after transplantation was the SF12-MCS, in which there was a statistically significant early improvement that did not meet the MCID threshold in the outpatient group ( $P$ value for change $=.001)$. However, early improvements in HRQL by SF12-MCS did exceed the MCID in the inpatient and ECMO groups ( $P$ value for change $\leq .002 ; P$ value 
TABLE 3. Effect estimates for change in health-related quality of life over time from before through 6 months after lung transplantation

\begin{tabular}{llcccc}
\hline \multicolumn{1}{c}{ Instrument } & Instrument type & ECMO group $(\mathbf{n}=\mathbf{1 7})$ & Inpatient group $(\mathbf{n}=\mathbf{4 8})$ & Outpatient group $(\mathbf{n}=\mathbf{1 2 4})$ & $\boldsymbol{P}$ value \\
\hline SF12-PCS (MCID = 5) & Generic-physical & $16.78(10.65-21.91)$ & $19.56(15.62-23.50)$ & $20.78(18.50-23.07)$ & .27 \\
SF12-MCS (MCID = 5) & Generic-mental & $8.78(3.31-14.26)$ & $7.48(3.97-10.99)$ & $4.48(2.47-6.49)$ \\
AQ20R (MCID = 1.75) & Respiratory-specific & $10.76(8.57-12.96)$ & $9.84(8.45-11.23)$ & $9.76(8.96-10.56)$ \\
EQ5D (MCID = 0.06) & Health utility & $0.31(0.20-0.42)$ & $0.29(0.22-0.36)$ & .01 \\
GDS (MCID = 1.65) & Depression scale & $4.81(3.15-6.48)$ & $3.43(2.38-4.49)$ & $0.17(0.13-0.21)$ \\
\hline
\end{tabular}

Data are presented as mean effect estimates with $95 \%$ confidence intervals as quantified by segmented linear mixed-effects models with a joint point at 6 months. Models were adjusted for age, sex, diagnosis, preoperative forced expiratory volume in 1 second, 6-minute walk distance, and Lung Allocation Score. Health-related quality of life instruments: generic physical instrument: SF12-PCS (Short Form 12-Physical Component Score), range 0 to 100; generic mental instrument: SF12-MCS (Short Form 12-Mental Component Score), range 0 to 100; respiratory-specific instrument: AQ20R (Airways Questionnaire 20-Revised), range 0 to 20, reverse-coded for analysis; health-utility instrument: EQ5D (EuroQoL 5D), range - 1.11 to 1; depression scale: GDS (Geriatric Depression Scale), range 0 to 15, reverse-coded for analysis. ECMO, Extracorporeal membrane oxygenation; SF12-PCS, short form 12-physical component score; MCID, minimally clinically important difference; SF12-MCS, short form 12-mental component score; AQ20R, airways questionnaire 20-revised; EQ5D, EuroQoL 5D; GDS, geriatric depression scale.

for difference among groups $=.01$ ). The effect estimates for change in HRQL when controlled for baseline GDS or in unadjusted analysis were not substantially different than the primary analysis (Tables E2 and E3). The effect estimate for change was also not substantially different when imputation methods were used for missing data (Table E4).

\section{DISCUSSION}

In a contemporary cohort of adults undergoing lung transplantation, we found that patients requiring ECMO as a bridge to transplantation experience substantial early improvements in HRQL and depressive symptoms that are comparable to those undergoing transplantation without bridging by ECMO. The improvements in generic-physical, respiratory-specific, and health utility metrics of HRQL, as well as depressive symptoms, exceeded by 1- to 5-fold what is considered the MCID. The greatest improvement was seen in respiratory-specific HRQL, whereas the smallest improvement was in generic-mental HRQL (SF12-MCS). Our findings are consistent with previous studies demonstrating either no or minimal improvement in HRQL after lung transplantation when using a generic-mental HRQL instrument. ${ }^{2,3}$ It remains unclear whether the reason for this improvement is attributable to high baseline scores or to the emergence of new problems after transplantation that negatively impact mental health-related quality of life, or whether the instrument itself is not sufficiently sensitive to detect changes in our population.

It is surprising that the subjects who were bridged to lung transplantation with ECMO reported similar improvements in HRQL and depressive symptoms as the other groups. Because surviving ECMO with resultant lung transplantation might be similar to surviving critical illness more generally, we hypothesized that HRQL and depressive symptoms would be worse in this group relative to others. Indeed, survivors of critical illness across medical and surgical populations report marked impairments in HRQL. ${ }^{19,20,23}$ Although this is speculative, differences between surviving general critical illness and the illness preceding lung transplantation might account for our findings. Generally, acute critical illness is an unexpected event, whereas most lung transplant recipients have suffered from advanced lung disease for some time. Thus, these patients may anticipate gradual, if not sudden, worsening. As a result, they may be more familiar with poor physical functioning, severe dyspnea, and the psychological challenges of potential loss of life. In addition, lung transplantation offers a potential reversal of chronic and progressive illness. This possibility may help patients mitigate the stresses of critical illness in a way that other critically ill patients cannot. Another possibility is that lung transplant recipients represent a group highly enriched to have strong social support. This support network can help mitigate the influence of illness on HRQL. Finally, lung transplant candidates are also not likely to have significant comorbidities because of the rigorous selection process, which may facilitate their recovery from critical illness.

To date, most studies evaluating success of ECMO as a bridge to lung transplantation have defined efficacy by survival alone. ${ }^{39-41}$ In our clinical experience, patients-even those on ECMO - are keenly interested in their potential quality of life after lung transplantation. Given the substantial potential for morbidity associated with ECMO and its increasing use as a bridge to transplantation, definitions of transplant efficacy should reflect the metrics most important to patients. Our study directly addresses this clinical need and complements the only other study of $\mathrm{HRQL}$ in recipients bridged to lung transplantation by ECMO. ${ }^{42}$ In that study, HRQL was assessed in 7 subjects bridged to transplantation with ECMO and 77 subjects who underwent transplantation without the use of ECMO bridging who survived the first postoperative year. HRQL 
in the non-ECMO group was assessed with a visual analog scale and, in the ECMO group, both a visual analog scale and the EQ5D. The investigators found no difference in HRQL between the 2 groups by the visual analog scale.

Our study has some limitations. Although our cohort represents the largest evaluation of the impact of lung transplantation on HRQL in patients bridged to transplantation with ECMO, our sample size is nonetheless small. The impact of this small sample size is reflected in the wide CIs surrounding the effect estimates of change in HRQL and depressive symptoms in the ECMO group. For example, in the SF12-PCS, the lower bound of the 95\% CI was nearly one-fold the MCID lower in the ECMO group compared with the inpatient and outpatient groups. Therefore, it is possible that lung transplantation for patients bridged on ECMO might not lead to improvements as great as those seen in the other groups. Despite this possible difference, patients bridged on ECMO still derive substantial benefits in addition to a clear survival benefit. Moreover, although the types of patients undergoing transplantation at our center are similar to many others, our findings might not be generalizable. Furthermore, HRQL outcomes and depressive symptoms beyond the first postoperative year were not addressed. Finally, although we chose a variety of HRQL instruments to perform a more comprehensive evaluation of HRQL, it is possible that some of the health domains impacted by ECMO, such as cognitive functioning, were not adequately queried. ${ }^{43,44}$

Despite these limitations, our study has several strengths. It is the first prospective longitudinal cohort study to evaluate the impact of lung transplantation on HRQL and depressive symptoms in patients who were bridged to transplantation with ECMO. Querying the same subjects before and repeatedly after transplantation provides the ability to evaluate changes in HRQL on an individual-subject level. Furthermore, our efforts to update preoperative assessments at the time of changes in clinical status and to address missing data through imputation approaches strengthen the validity of our effect estimates. By using 4 instruments to assess HRQL as well as a depression scale and using 2 comparison groups, we were able to provide a more comprehensive picture of HRQL after transplantation in those on ECMO in the run-up to transplantation. Finally, the fact that our study required 7 years to amass 17 subjects bridged to transplantation with ECMO with posttransplantation follow-up suggests that larger sample sizes are likely to result only from multicenter studies or efforts from high ECMO volume centers.

\section{CONCLUSIONS}

In conclusion, our findings show that the impact of lung transplantation on HRQL and depressive symptoms for patients requiring preoperative ECMO is large and appears to be similar to that for noncritically ill candidates for transplantation. This knowledge may provide clinicians with more tools to counsel critically ill patients and their families when considering ECMO as a bridge to transplantation. Future work should focus on a multicenter approach to measuring $\mathrm{HRQL}$ and investigate whether our findings apply to the international transplantation community.

\section{Conflict of Interest Statement}

Authors have nothing to disclose with regard to commercial support.

\section{References}

1. Weill D, Benden C, Corris PA, Dark JH, Davis RD, Keshavjee S, et al. A consensus document for the selection of lung transplant candidates: 2014: an update from the pulmonary transplantation council of the international society for heart and lung transplantation. J Heart Lung Transplant. 2015; 34:1-15.

2. Singer LG, Chowdhury NA, Faughnan ME, Granton J, Keshavjee S, Marras TK, et al. Effects of recipient age and diagnosis on health-related quality-of-life benefit of lung transplantation. Am J Respir Crit Care Med. 2015;192:965-73.

3. Singer JP, Katz PP, Soong A, Shrestha P, Huang D, Ho J, et al. Effect of lung transplantation on health-related quality of life in the era of the lung allocation score: a US prospective cohort study. Am J Transplant. 2017;17:1334-45.

4. Gottlieb J, Warnecke G, Hadem J, Dierich M, Wiesner O, Fühner T, et al. Outcome of critically ill lung transplant candidates on invasive respiratory support. Intensive Care Med. 2012;38:968-75.

5. Kilic A, Beaty CA, Merlo CA, Conte JV, Shah AS. Functional status is highly predictive of outcomes after redo lung transplantation: an analysis of 390 cases in the modern era. Ann Thorac Surg. 2013;96:1804-11; discussion 1811.

6. Moreno P, Alvarez A, Santos F, Vaquero JM, Baamonde C, Redel J, et al. Extended recipients but not extended donors are associated with poor outcomes following lung transplantation. Eur J Cardiothorac Surg. 2014;45:1040-7.

7. Mason DP, Thuita L, Nowicki ER, Murthy SC, Pettersson GB, Blackstone EH. Should lung transplantation be performed for patients on mechanical respiratory support? The US experience. J Thorac Cardiovasc Surg. 2010;139:765-73.e1.

8. Chiumello D, Coppola S, Froio S, Colombo A, Del Sorbo L. Extracorporeal life support as bridge to lung transplantation: a systematic review. Crit Care. 2015 19:19.

9. Diaz-Guzman E, Hoopes CW, Zwischenberger JB. The evolution of extracorporeal life support as a bridge to lung transplantation. ASAIO J. 2013;59:3-10.

10. Hayanga JWA, Lira A, Aboagye JK, Hayanga HK, D’Cunha J. Extracorporeal membrane oxygenation as a bridge to lung transplantation: what lessons migh we learn from volume and expertise? Interact Cardiovasc Thorac Surg. 2016; 22:406-10.

11. Toyoda Y, Bhama JK, Shigemura N, Zaldonis D, Pilewski J, Crespo M, et al. Efficacy of extracorporeal membrane oxygenation as a bridge to lung transplantation. J Thorac Cardiovasc Surg. 2013;145:1065-70; discussion 1070-1.

12. Todd EM, Biswas Roy S, Hashimi AS, Serrone R, Panchanathan R, Kang P, et al. Extracorporeal membrane oxygenation as a bridge to lung transplantation: a single-center experience in the present era. J Thorac Cardiovasc Surg. 2017 154:1798-809.

13. Hoetzenecker K, Donahoe L, Yeung JC, Azad S, Fan E, Ferguson ND, et al. Extracorporeal life support as a bridge to lung transplantation-experience of a highvolume transplant center. J Thorac Cardiovasc Surg. 2018;155:1316-28.e1.

14. Hoopes CW, Kukreja J, Golden J, Davenport DL, Diaz-Guzman E Zwischenberger JB. Extracorporeal membrane oxygenation as a bridge to pulmonary transplantation. J Thorac Cardiovasc Surg. 2013;145:862-7; discussion 867-8.

15. Banga A, Mohanka M, Mullins J, Bollineni S, Kaza V, Ring S, et al. Hospital length of stay after lung transplantation: independent predictors and association with early and late survival. J Heart Lung Transplant. 2017;36:289-96.

16. Lansink-Hartgring AO, van den Hengel B, van der Bij W, Erasmus ME, Mariani MA, Rienstra M, et al. Hospital costs of extracorporeal life support therapy. Crit Care Med. 2016;44:717-23.

17. Inci I, Klinzing S, Schneiter D, Schuepbach RA, Kestenholz P, Hillinger S, et al Outcome of extracorporeal membrane oxygenation as a bridge to lung transplantation: an institutional experience and literature review. Transplantation. 2015; 99:1667-71 
18. Makdisi G, Wang IW. Extracorporeal membrane oxygenation (ECMO): review of a lifesaving technology. J Thorac Dis. 2015;7:E166-76.

19. McNelly AS, Rawal J, Shrikrishna D, Hopkinson NS, Moxham J, Harridge SD, et al. An exploratory study of long-term outcome measures in critical illness survivors: construct validity of physical activity, frailty, and health-related quality of life measures. Crit Care Med. 2016;44:e362-9.

20. Wolters AE, Peelen LM, Welling MC, Kok L, de Lange DW, Cremer OL, et al. Long-term mental health problems after delirium in the ICU. Crit Care Med. 2016;44:1808-13.

21. Fleck TP, Dangel G, Bächle F, Benk C, Grohmann J, Kroll J, et al. Long-term follow-up on health-related quality of life after mechanical circulatory support in children. Pediatr Crit Care Med. 2017;18:176-82.

22. Chandler HK, Teppa B, Johnson KA, McCracken C, Fortenberry JD, Paden ML. Determining comorbidities and quality of life among pediatric survivors of extracorporeal life support. J Crit Care. 2015;30:1085-9.

23. Schmidt M, Zogheib E, Rozé H, Repesse X, Lebreton G, Luyt CE, et al. The PRESERVE mortality risk score and analysis of long-term outcomes after extracorporeal membrane oxygenation for severe acute respiratory distress syndrome. Intensive Care Med. 2013;39:1704-13.

24. Kolaitis NA, Huang D, Soong A, Shrestha P, Golden J, Leard LE, et al. ECMO prior to lung transplantation does not impact health-related quality of life (HRQL) after transplant. J Heart Lung Transplant. 2017;36: S72-3.

25. Singer JP, Chen J, Blanc PD, Leard LE, Kukreja J, Chen H. A thematic analysis of quality of life in lung transplant: the existing evidence and implications for future directions. Am J Transplant. 2013;13:839-50.

26. Seiler A, Klaghofer R, Ture M, Komossa K, Martin-Soelch C, Jenewein J. A systematic review of health-related quality of life and psychological outcomes after lung transplantation. J Heart Lung Transplant. 2016;35:195-202.

27. Ware J Jr, Kosinski M, Keller SD. A 12-item Short-Form Health Survey: construction of scales and preliminary tests of reliability and validity. Med Care. 1996;34:220-33.

28. Beaton DE, Boers M, Wells GA. Many faces of the minimal clinically important difference (MCID): a literature review and directions for future research. Curr Opin Rheumatol. 2002;14:109-14.

29. Chen H, Eisner MD, Katz PP, Yelin EH, Blanc PD. Measuring disease-specific quality of life in obstructive airway disease: validation of a modified version of the airways questionnaire 20. Chest. 2006;129:1644-52.

30. Norman GR, Sloan JA, Wyrwich KW. Interpretation of changes in health-related quality of life: the remarkable universality of half a standard deviation. Med Care. 2003;41:582-92.

31. EuroQol Group. EuroQol-a new facility for the measurement of health-related quality of life. Health Policy. 1990;16:199-208.
32. Dew MA, Rosenberger EM, Myaskovsky L, DiMartini AF, DeVito Dabbs AJ, Posluszny DM, et al. Depression and anxiety as risk factors for morbidity and mortality after organ transplantation: a systematic review and meta-analysis. Transplantation. 2015;100:988-1003.

33. Smith PJ, Blumenthal JA, Snyder LD, Mathew JP, Durheim MT, Hoffman BM, et al. Depressive symptoms and early mortality following lung transplantation: a pilot study. Clin Transplant. 2017;31:e12874.

34. Davydow DS, Gifford JM, Desai SV, Bienvenu OJ, Needham DM. Depression in general intensive care unit survivors: a systematic review. Intensive Care Med. 2009;35:796-809.

35. Yesavage JA, Brink TL, Rose TL, Lum O, Huang V, Adey M, et al. Development and validation of a geriatric depression screening scale: a preliminary report. $J$ Psychiatr Res. 1982-1983;17:37-49.

36. Egan TM, Murray S, Bustami RT, Shearon TH, McCullough KP, Edwards LB, et al. Development of the new lung allocation system in the United States. Am J Transplant. 2006;6(5 Pt 2):1212-27.

37. Laird NM. Missing data in longitudinal studies. Stat Med. 1988;7:305-15.

38. Lee KJ, Simpson JA. Introduction to multiple imputation for dealing with missing data. Respirology. 2014;19:162-7.

39. Biscotti M, Gannon WD, Agerstrand C, Abrams D, Sonett J, Brodie D, et al. Awake extracorporeal membrane oxygenation as bridge to lung transplantation: a 9-year experience. Ann Thorac Surg. 2017;104:412-9.

40. Schechter MA, Ganapathi AM, Englum BR, Speicher PJ, Daneshmand MA, Davis RD, et al. Spontaneously breathing extracorporeal membrane oxygenation support provides the optimal bridge to lung transplantation. Transplantation. 2016;100:2699-704.

41. Hayes D Jr, Tobias JD, Tumin D. Center volume and extracorporeal membrane oxygenation support at lung transplantation in the lung allocation score era. Am J Respir Crit Care Med. 2016;194:317-26.

42. Lansink-Hartgring AO, van der Bij W, Verschuuren EA, Erasmus ME, de Vries AJ, Vermeulen KM, et al. Extracorporeal life support as a bridge to lung transplantation: a single-center experience with an emphasis on health-related quality of life. Respir Care. 2017;62:588-94.

43. Smith PJ, Blumenthal JA, Carney RM, Freedland KE, O'Hayer CVF, Trulock EP, et al. Neurobehavioral functioning and survival following lung transplantation. Chest. 2014;145:604-11.

44. Smith PJ, Rivelli S, Waters A, Reynolds J, Hoyle A, Flowers M, et al. Neurocognitive changes after lung transplantation. Ann Am Thorac Soc. 2014;11: $1520-7$.

Key Words: ECMO, lung transplantation, HRQL, quality of life, QOL, HRQoL, health-related quality of live, extracorporeal membrane oxygenation 
TABLE E1. Instruments used to measure health-related quality of life and depressive symptoms

\begin{tabular}{|c|c|c|c|c|}
\hline Instrument & Instrument type & Questions & Range & MCID \\
\hline SF12-PCS & Generic-physical & Physical functioning, physical role, bodily pain, general health & $0-100$ & 5 \\
\hline SF12-MCS & Generic-mental & Vitality, social functioning, emotional function, mental health & $0-100$ & 5 \\
\hline AQ20R & Respiratory-specific & Impact of breathing on life & $0-20^{*}$ & $1.75 \dagger$ \\
\hline EQ5D & Utility & Mobility, self-care, pain, anxiety & $-1.11-1$ & 0.06 \\
\hline GDS & Mental & Depression, mental health & $0-30^{*}$ & $1.65 \dagger$ \\
\hline
\end{tabular}

Health-related quality of life instruments: generic physical instrument: SF12-PCS (Short Form 12-Physical Component Score), range 0 to 100; generic mental instrument: SF12MCS (Short Form 12-Mental Component Score), range 0 to 100; respiratory-specific instrument: AQ20R (Airways Questionnaire 20-Revised), range 0 to 20, reverse-coded for analysis; health-utility instrument: EQ5D (EuroQoL 5D), range -1.11 to 1; depression scale: GDS (Geriatric Depression Scale), range 0 to 15, reverse-coded for analysis. $M C I D$, Minimally clinically important difference; SF12-PCS, short form 12-physical component score; SF12-MCS, short form 12-mental component score; AQ20R, airways questionnaire 20-revised; EQ5D, EuroQoL 5D;GDS, geriatric depression scale. *Reverse-coded for analysis. $\dagger 0.5$ standard deviation used in lieu of established minimally clinically important difference.

TABLE E2. Effect estimates for change in health-related quality of life over time from before through 6 months after lung transplantation, controlling for baseline depressive symptoms

\begin{tabular}{llcccc}
\hline \multicolumn{1}{c}{ Instrument } & Instrument type & ECMO group $(\mathbf{n}=\mathbf{1 7})$ & Inpatient group $(\mathbf{n}=48)$ & Outpatient group $(\mathbf{n}=\mathbf{1 2 4})$ & $\boldsymbol{P}$ value \\
\hline SF12-PCS (MCID = 5) & Generic-physical & $16.63(10.35-22.91)$ & $19.65(15.56-23.74)$ & $20.98(18.68-23.27)$ & .47 \\
SF12-MCS (MCID = 5) & Generic-mental & $8.68(3.06-14.30)$ & $7.84(4.19-11.49)$ & $4.42(2.38-6.45)$ \\
AQ20R (MCID = 1.75) & Respiratory-specific & $10.79(8.57-13.01)$ & $10.13(8.69-11.57)$ & $9.85(9.04-10.65)$ \\
EQ5D (MCID = 0.06) & Health utility & $0.30(0.19-0.42)$ & $0.30(0.23-0.38)$ & .01 \\
GDS (MCID = 1.65) & Depression scale & $4.80(3.23-6.38)$ & $3.62(2.59-4.65)$ & .67 \\
\hline
\end{tabular}

Data are presented as mean effect estimates with $95 \%$ confidence intervals as quantified by segmented linear mixed-effects models with a joint point at 6 months. Models were adjusted for age, sex, diagnosis, preoperative forced expiratory volume in 1 second, 6-minute walk distance, lung allocation score, and pretransplantation Geriatric Depression Scale score. Health-related quality of life instruments: generic physical instrument: SF12-PCS (Short Form 12-Physical Component Score), range 0 to 100; generic mental instrument: SF12-MCS (Short Form 12-Mental Component Score), range 0 to 100; respiratory-specific instrument: AQ20R (Airways Questionnaire 20-Revised), range 0 to 20, reverse-coded for analysis; health-utility instrument: EQ5D (EuroQoL 5D), range -1.11 to 1; depression scale: GDS (Geriatric Depression Scale), range 0 to 15, reverse-coded for analysis. ECMO, Extracorporeal membrane oxygenation; SF12-PCS, short form 12-physical component score; $M C I D$, minimally clinically important difference; SF12-MCS, short form 12-mental component score; $A Q 20 R$, airways questionnaire 20-revised; EQ5D, EuroQoL 5D; GDS, geriatric depression scale. 
TABLE E3. Unadjusted effect estimates for change in health-related quality of life over time from before through 6 months after lung transplantation

\begin{tabular}{llcccc}
\hline \multicolumn{1}{c}{ Instrument } & Instrument type & ECMO group $(\mathbf{n}=\mathbf{1 7})$ & Inpatient group $(\mathbf{n}=48)$ & Outpatient group $(\mathbf{n}=\mathbf{1 2 4})$ & $\boldsymbol{P}$ value \\
\hline SF12-PCS (MCID = 5) & Generic-physical & $16.66(10.65-22.66)$ & $19.70(15.86-22.75)$ & $20.92(18.64-23.19)$ \\
SF12-MCS (MCID = 5) & Generic-mental & $8.87(3.47-14.26)$ & $7.75(4.31-11.19)$ & $4.40(2.41-6.40)$ \\
AQ20R (MCID = 1.75) & Respiratory-specific & $10.45(8.31-12.60)$ & $9.85(8.50-11.21)$ & $9.78(8.99-10.57)$ \\
EQ5D (MCID = 0.06) & Health utility & $0.31(0.20-0.42)$ & $0.29(0.22-0.36)$ & .02 \\
GDS (MCID = 1.65) & Depression scale & $4.75(3.11-6.38)$ & $3.45(2.42-4.48)$ & .63 \\
\hline
\end{tabular}

Data are presented as mean effect estimates with $95 \%$ confidence intervals as quantified by segmented linear mixed-effects models with a joint point at 6 months. Models are unadjusted. Health-related quality of life instruments: generic physical instrument: SF12-PCS (Short Form 12-Physical Component Score), range 0 to 100; generic mental instrument: SF12-MCS (Short Form 12-Mental Component Score), range 0 to 100; respiratory-specific instrument: AQ20R (Airways Questionnaire 20-Revised), range 0 to 20, reverse-coded for analysis; health-utility instrument: EQ5D (EuroQoL 5D), range -1.11 to 1; depression scale: GDS (Geriatric Depression Scale), range 0 to 15 , reverse-coded for analysis. ECMO, Extracorporeal membrane oxygenation; SF12-PCS, short form 12-physical component score; MCID, minimally clinically important difference; SF12-MCS, short form 12-mental component score; $A Q 20 R$, airways questionnaire 20-revised; EQ5D, EuroQoL 5D; GDS, geriatric depression scale.

TABLE E4. Effect estimates for change in health-related quality of life over time from before through 6 months after lung transplantation with imputed data

\begin{tabular}{|c|c|c|c|c|c|}
\hline Instrument & Instrument type & ECMO group $(n=17)$ & Inpatient group $(n=48)$ & Outpatient group $(n=124)$ & $P$ value \\
\hline SF12-PCS (MCID = 5) & Generic-physical & $17.41(11.37-23.46)$ & $18.50(14.57-22.42)$ & $19.54(17.18-21.90)$ & .44 \\
\hline SF12-MCS $(\mathrm{MCID}=5)$ & Generic-mental & $9.10(3.96-14.24)$ & $7.52(4.17-10.87)$ & $3.86(1.86-5.87)$ & .02 \\
\hline AQ20R $(\mathrm{MCID}=1.75)$ & Respiratory-specific & $10.54(8.18-12.91)$ & $9.28(7.78-10.78)$ & $9.10(8.20-10.00)$ & .72 \\
\hline EQ5D $(\mathrm{MCID}=0.06)$ & Health utility & $0.29(0.18-0.40)$ & $0.25(0.19-0.33)$ & $0.15(0.11-0.19)$ & .004 \\
\hline GDS $(\mathrm{MCID}=1.65)$ & Depression scale & $4.59(2.95-6.22)$ & $3.33(2.28-4.37)$ & $3.26(2.64-3.89)$ & .28 \\
\hline
\end{tabular}

Data missing at random were imputed with 10-fold multiple imputation. Data missing due to subject illness were imputed using the median of the lowest quartile of health-related quality of life scores for all other participants at that time point. Data are presented as mean effect estimates with $95 \%$ confidence intervals as quantified by segmented linear mixed-effects models with a joint point at 6 months. Models were adjusted for age, sex, diagnosis, preoperative forced expiratory volume in 1 second, 6 -minute walk distance, and Lung Allocation Score. Health-related quality of life instruments: generic physical instrument: SF12-PCS (Short Form 12-Physical Component Score), range 0 to 100; generic mental instrument: SF12-MCS (Short Form 12-Mental Component Score), range 0 to 100; respiratory-specific instrument: AQ20R (Airways Questionnaire 20-Revised), range 0 to 20, reverse-coded for analysis; health-utility instrument: EQ5D (EuroQoL 5D), range -1.11 to 1; depression scale: GDS (Geriatric Depression Scale), range 0 to 15, reversecoded for analysis. ECMO, Extracorporeal membrane oxygenation; SF12-PCS, short form 12-physical component score; $M C I D$, minimally clinically important difference; SF12-MCS, short form 12-mental component score; AQ20R, airways questionnaire 20-revised; EQ5D, EuroQoL 5D; GDS, geriatric depression scale. 